\title{
Rapid Prototyping of Microfluidics Devices using Xurograhy Method
}

\author{
Fiona W.M. Ling ${ }^{1 *}$, Wafaa K. Mahmood ${ }^{1}$ and Hayder A. Abdulbari ${ }^{1}$ \\ ${ }^{1}$ Centre of Excellence for Advanced Research in Fluid Flow, Universiti Malaysia Pahang, Lebuhraya Tun Razak 26300 Kuantan, \\ Pahang, Malaysia
}

\begin{abstract}
Rapid prototyping of microchannel gain lots of attention from researchers along with the rapid development of microfluidic technology. The conventional methods carried few disadvantages such as high cost, time consuming, required high operating pressure and temperature and involve expertise in operating the equipment. In this work, new method adapting xurography method is introduced to replace the conventional method of fabrication of microchannels. The novelty in this study is replacing the adhesion film with clear plastic film which was used to cut the design of the microchannel as the material is more suitable for fabricating more complex microchannel design. The microchannel was then mold using polymethyldisiloxane (PDMS) and bonded with a clean glass to produce a close microchannel. The microchannel produced had a clean edge indicating good master mold was produced using the cutting plotter and the bonding between the PDMS and glass was good where no leakage was observed. The materials used in this method is cheap and the total time consumed is less than 5 hours where this method is suitable for rapid prototyping of microchannel.
\end{abstract}

\section{Introduction}

Microfluidics technology involves the utilization of the devices that have the dimensions less than $1 \mathrm{~mm}$ which allow the precision in handling of fluids. This technology have been developed tremendously and have massive impact in wide variety of applications. Low sample and reagent volume is use due to the usage of micrometer-sized channel resulting in creating less waste and environmental problems. The miniaturization of analytical technique using microfluidic technology bring advantages such as reduce the equipment size, shorten the reaction or analysis time and the possibility of develop of portable devices.

The miniaturization system has provide a new platform to fluids manipulation components [1] such as pumps, valves, filters and mixing. Microfluidic devices were also widely used in reaction [2-4] and heat transfer $[5,6]$. Microfluidic plays an important role in detection technique including separation [7] and food analysis [8, 9]. Besides, lab-on-chips were greatly incorporated in clinical [10-12], cell biology [13] and droplet generators $[14,15]$.

Rapid prototyping of microfluidic devices have been the concern of the researchers along with the instantaneous development of microfluidic technique nowadays. Several rapid prototyping methods to fabricate microchannels were introduced including wet etching, conventional machining [16], photolithography, soft lithography, hot embossing, injection molding, laser ablation, and plasma etching [17].
There are some disadvantages of these conventional methods. Machining itself involve combination of few techniques of microchannels fabrications such as wet etching, photolithography and others which also requires the clean room facilities thus increases the cost of fabrication [18-20]. Embossing process consumes lots of time [21] and it is not suitable for prototyping few microchannels for testing purposes. Due to the complexity of molding equipment and high operating temperature and pressure [22], injection molding is more suitable for industrial compare to research application.

In most of the laboratory environment, soft lithography is used as rapid prototyping due to the advantages such as low cost and less time consuming $[23,24]$. The material use in soft lithography is polydimethylsiloxane (PDMS) which has high optical transparency and high gas permeability thus suitable even for cell and biology applications [25]. Due to the elastomeric properties of PDMS even complex 3D microchannels also can be fabricated [26]. PDMS also showed excellent bonding to itself or other materials [27]. However, soft lithography involving fabrication of special silicon master composed of photoresist with the design of microchannel on it. This process is much more consuming time and need special equipment also chemicals especially when changes of design required.

With the development of the cutting plotter nowadays, the resolution and alignment of cutting plotter have drastically improved thus lead to microscale prototyping [28]. Xurography is a method where a master mold is cut using a cutting plotter machine.

\footnotetext{
* Corresponding author: fionalingwm $@$ gmail.com
} 
Martínez-López et al. [29] and Neuville et al. [30] utilized adhesive film to cut the master mold. However, the produced master mold from adhesive film is suitable for simple design where it is hard to manipulate when complicated design is required. In order to solve the problems occur when using the conventional method, therefore in this study, new low-cost rapid fabrication of microchannel adapting xurography method is introduced. Clear plastic film is chosen in this study, replacing adhesive films, to cut the desired design.

\section{Methodology}

Semi-clogged heart blood vessels (Fig 1a) according to the angiogram of the real heart vessels (Fig 1b) was first drawn using Graphtec Studio. A clear plastic film with the thickness around $200 \mu \mathrm{m}$ was used to cut the master mold using Graphtec cutting plotter CE6000-60 (Fig 2a). The design was peel off from the plastic film (Fig 2b) and pasted on glass (Fig 2c) using a thin layer of heat resistance glue. Glass was chosen due to its chemical resistance property.

Mixture of PDMS and curing agent at the ratio of 10:1 was prepared in a disposable plastic cup. $60 \mathrm{~g}$ of PDMS and $10 \mathrm{~g}$ of curing agent were weighed and then mixed using a mixing machine (model: Thinky mixer). The revolution balance dial was adjusted according to the total weight of the cup and the adapter of the mixer. The program was set to mix the mixture at $1000 \mathrm{rpm}$ for $5 \mathrm{~min}$ and defoaming (degassing) at $400 \mathrm{rpm}$ for another 5 min.

The degassed mixture was then poured on the design (Fig 2d) which placed in a petri dish which was then placed in a desiccator for $40 \mathrm{~min}$ to remove all the air bubbles that might trapped inside (Fig 2e). The PDMS was then placed in an oven at $60{ }^{\circ} \mathrm{C}$ for 2 and a half hours for soft baking process which can solidify the PDMS (Fig 2f).

The PDMS was cut along the edge of the glass using a scalpel (surgery knife) then peeled off carefully and slowly from the design using a tweezer (Fig 2g). The inlet and outlet of the microchannel were punched using a puncher (Fig 2h). Both the PDMS and glass slide were placed in plasma cleaner for 2 minutes (Fig 2i) and then put together where the design of microchannel facing the glass slide and pressed slightly to let them bond together (Fig 2j). A suitable length of tubes was cut, and all the inlet and outlets were connected using the tubes. The microchannel was ready for further testing.

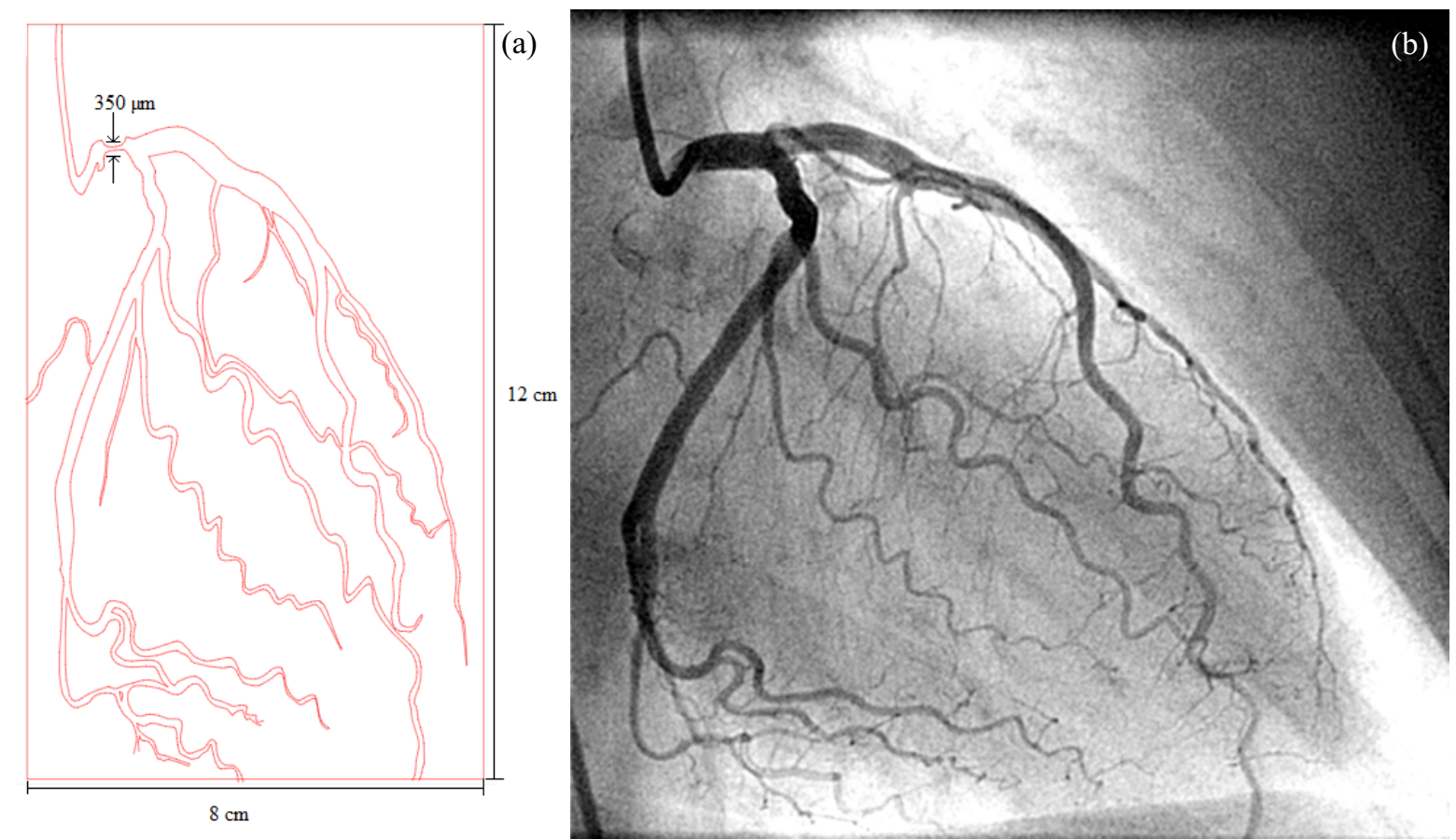

Fig 1. (a) Design of the microchannel using Graphtec Studio (b) Angiogram of heart blood vessels 


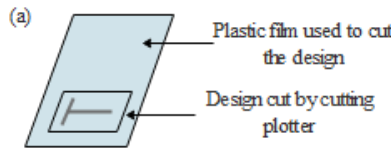

(b)

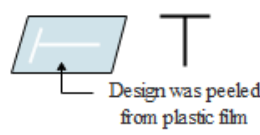

(c)

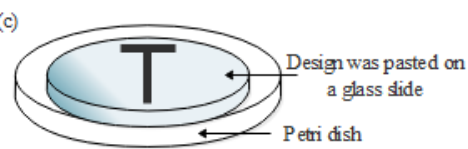

(d)

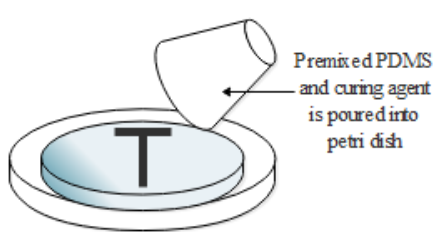

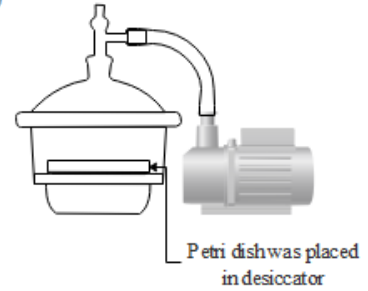

(f)
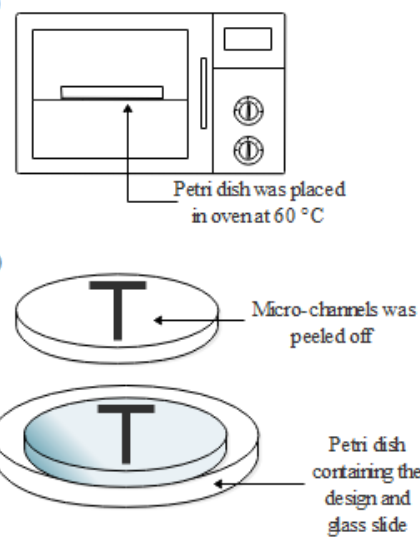

(h)

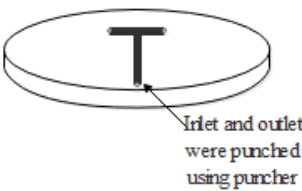

(i)

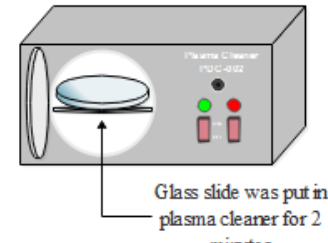

(j)

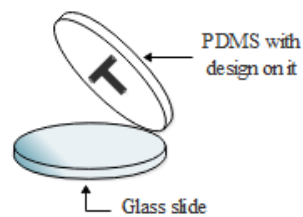

Fig 2. Schematic diagram depicting xurography method

\section{Results and Discussions}

The cutting quality of the cutting plotter is depended on some parameters such as the sharpness of cutting blade, cutting force, cutting speed, the condition of the cutting area and the material used. It is advisable to test cut for cutting quality and setting of the cutting plotter checking. For a more complicated design, it is always recommended that use a lower speed for a better cutting quality especially for a design having curvy edges. For a thicker material, the higher cut force will be needed. However, test cut start from a lower cut force is necessary to prevent damage to both cutting blade and cutting area of the cutting plotter where the penetration of the blade should not exceed the material itself. It is also suggested not to use the maximum cut force. The material use should always be compatible with the specification of the blade to longer the lifespan of the blade. In this case, a clean and high-quality cut is required. Thus the cut speed was set at a lower level in contrast to the higher level of cut force was set since the thick material was used.

Qualitative visualization of the cross-sectional of the microchannel was done to evaluate the quality of the produced master mold (clear plastic film containing design) which would directly affect the quality of microchannel produced. A small part of the straight microchannel was cut and brought to a scanning electron microscope (SEM) for inspection purpose. Straight microchannel was selected for clearer visualization. As shown in Fig 3, clean edge of microchannel can be observed at the cross-sectional area and along the entire straight channel which indicated that high quality of master mold was produced. This result also indicated that the resolution of the cutting plotter and the sharpness of the cutting blade were good enough to produce the master mold as it did not produce master mold with crooked edge which would then affect the quality of the produced microchannel. As a result, this method can be used in rapid prototyping of microchannel as high quality of master mold can be produced.

The complete microchannel was then brought for testing by flowing liquid across it. This process was done to test whether there is any leakage especially at inlet and outlet of the microchannel. Besides, by flowing liquid across the microchannel, checking for the adhesion of the PDMS on glass also can be done. Green food dye was used for the ease of visual inspection. The flow of the fluid was provided by the vacuum and pressure controller (model: Elveflow OB1) with a pressure set of 100-500 mbar to push the fluid out from the reservoir. Different pressure was set to check the maximum volume of fluid can be carried by the microchannel before backflow of liquid happen. In this study, the maximum pressure set is around $450 \mathrm{mbar}$ where the flow rate of the fluid is around $3000 \mu \mathrm{l} / \mathrm{min}$. From Fig 4, there was no any leakage at either the inlet, outlets or along the microchannel even when the pressure was set at higher pressure, in this case 500 mbar. The result indicated that the bonding between the PDMS and the glass was good. It is expected that the great bonding between these two surfaces can withstand higher pressure and flow rate in the channel without affecting the bonding. This quality is advantageous for researchers who required higher pressure and flow rate across the channel for their work.

By stimulating the real blood vessels, researchers can use the microchannels instead of animal testing which did not follow the ethics of life. Beside heart vessels, other blood vessels also can be stimulated using this method. By using microchannel, the time consuming for the experiment is believed to significantly reduced 
compared to animal testing. Thus, this method of fabrication of microchannel will play a significant role in the development of clinical and drug delivery studies.

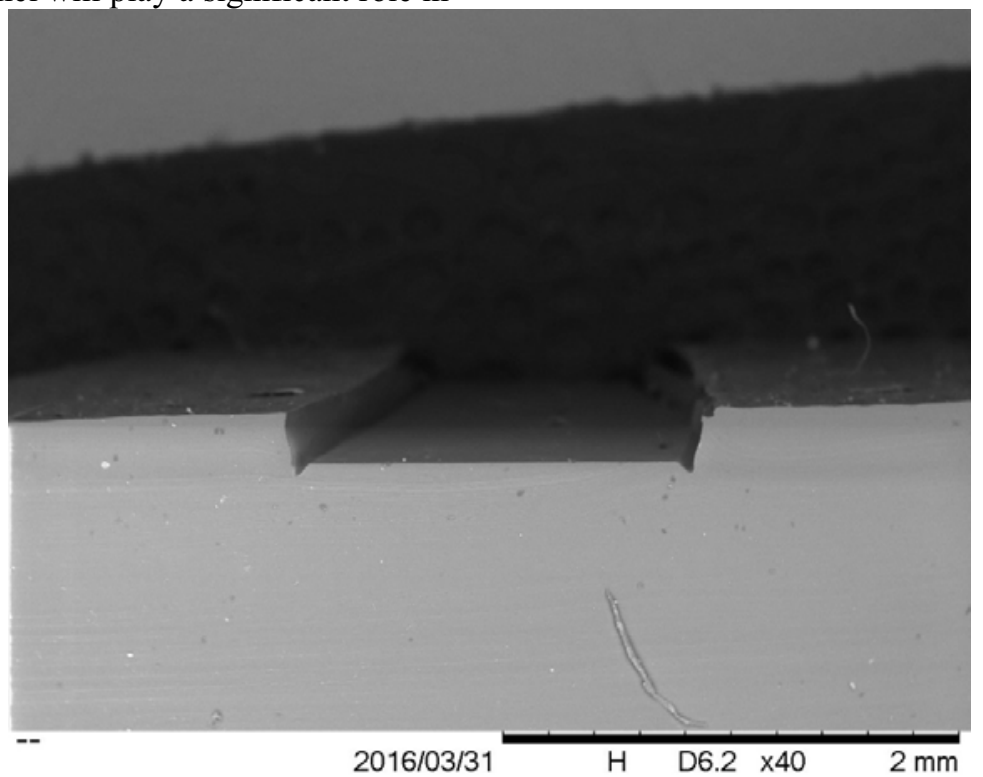

Fig 3. SEM image of cross-section of microchannel showing the straight channel. The edge of the microchannel was clean indicating high quality of the master mold was produced.

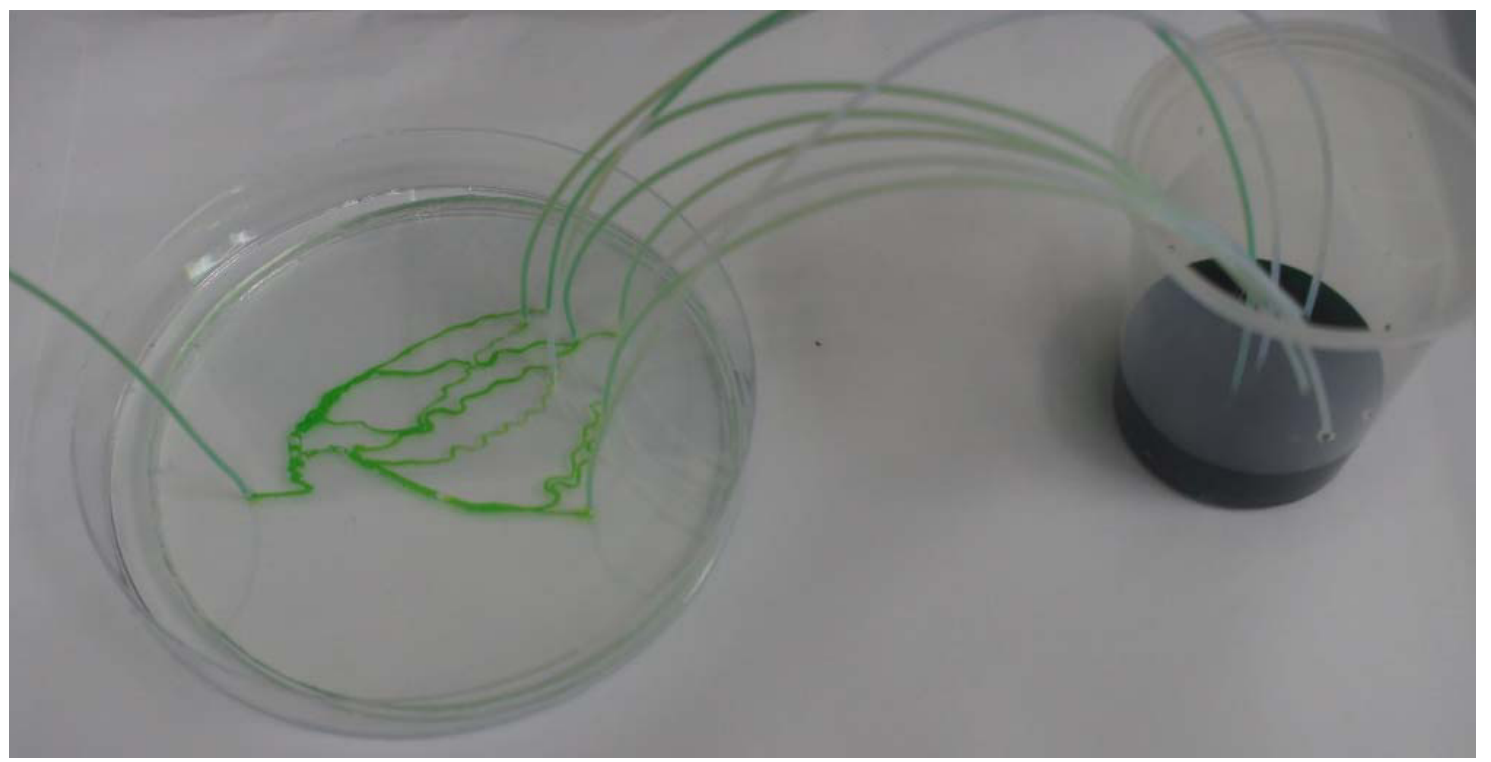

Fig 4. The complete microchannel was brought for flow testing. Food dye was used for visual inspection of leakage testing of the complete microchannel. The bonding of the PDMS and glass was good and no leakage was observed.

\section{Conclusions}

In this study presented a rapid and low-cost fabrication of microchannel using xurography method where the fabrication process only took less than 5 hours and there is no need expertise to operate all the equipment. The quality of the microchannel was depended on the cutting quality of the master mold where high quality of the cutting would produce a high-quality microchannel with clean edges. The thickness of the microchannel is depending on the paper material that used to cut the design using a cutting plotter. The current method is currently useful in producing complicated design such as blood streams that is useful in clinical researches thus replacing animal testing. The feasibility of using other materials and the ability to cut blade to cut even smaller dimension should be further explored to develop this method to other fields.

\section{References}

1. Fiorini, G.S. and D.T. Chiu, Disposable microfluidic devices: Fabrication, function, and application. BioTechniques 2005. 38: p. 429-446.

2. deMello, A.J., Control and detection of chemical reactions in microfluidic systems. Nature, 2006. 442(7101): p. 394-402. 
3. Truter, L.A., et al., The application of palladium and zeolite incorporated chipbased microreactors. Applied Catalysis A: General, 2016. 515: p. 72-82.

4. Ying, X., et al., Efficient Fischer-Tropsch microreactor with innovative aluminizing pretreatment on stainless steel substrate for Co/Al2O3 catalyst coating. Fuel Processing Technology, 2016. 143: p. 51-59.

5. Morgan, A.J.L., et al., Efficient microwave heating of microfluidic systems. Sensors and Actuators B: Chemical, 2013. 181: p. 904909.

6. Lee, S.H. and I. Mudawar, Investigation of flow boiling in large micro-channel heat exchangers in a refrigeration loop for space applications. International Journal of Heat and Mass Transfer, 2016. 97: p. 110-129.

7. Nam, Y., M. Kim, and T. Kim, Pneumatically controlled multi-level microchannel for separation and extraction of microparticles. Sensors and Actuators B: Chemical, 2014. 190: p. 86-92.

8. Guo, L., et al., Application of microfluidic "lab-on-a-chip" for the detection of mycotoxins in foods. Trends in Food Science \& Technology, 2015. 46(2, Part A): p. 252263.

9. Muijlwijk, K., C. Berton-Carabin, and K. Schroën, Cross-flow microfluidic emulsification from a food perspective. Trends in Food Science \& Technology, 2016. 49: p. 51-63.

10. Boussommier-Calleja, A., et al., Microfluidics: A new tool for modeling cancer-immune interactions. Trends in Cancer, 2016. 2(1): p. 6-19.

11. Kozminsky, M., Y. Wang, and S. Nagrath, The incorporation of microfluidics into circulating tumor cell isolation for clinical applications. Current Opinion in Chemical Engineering, 2016. 11: p. 59-66.

12. Tay, A., et al., Advances in microfluidics in combating infectious diseases. Biotechnology Advances, 2016. 34(4): p. 404-421.

13. Grünberger, A., W. Wiechert, and D. Kohlheyer, Single-cell microfluidics: opportunity for bioprocess development. Current Opinion in Biotechnology, 2014. 29: p. 15-23.

14. Zhu, Y. and Q. Fang, Analytical detection techniques for droplet microfluidics-A review. Analytica Chimica Acta, 2013. 787: p. 24-35.

15. Shah, R.K., et al., Designer emulsions using microfluidics. Materials Today, 2008. 11(4): p. 18-27.

16. T., S., et al., Microstructure grooves with a width of less than 50 [mu]m cut with ground hard metal micro end mills. Precision Engineering, 1999. 23: p. 229-235.
17. Rossier, J.S., et al., Plasma etched polymer microelectrochemical systems. Lab Chip, 2002. 2(3): p. 145-50.

18. Martynova, L., et al., Fabrication of plastic microfluid channels by imprinting methods. Analytical Chemistry, 1997. 69(23): p. 47839.

19. $\mathrm{Xu}, \mathrm{J}$., et al., Room-temperature imprinting method for plastic microchannel fabrication. Analytical Chemistry, 2000. 72(8): p. 1930-3.

20. Rodriguez, I., et al., Rapid prototyping of glass microchannels. Analytica Chimica Acta, 2003. 496(1-2): p. 205-215.

21. Tsao, C.-W., et al., Rapid polymer microchannel fabrication by hot roller embossing process. Microsystem Technologies, 2012. 18(6): p. 713-722.

22. Çetin, B., A.K. Koska, and M. Erdal, Warpage characterization of microchannels fabricated by injection molding. Journal of Micro and Nano-Manufacturing, 2015. 3(2): p. 021005-021005.

23. Friend, J. and L. Yeo, Fabrication of Microfluidic Devices using Polydimethylsiloxane. Biomicrofluidics, 2010. 4.

24. Martin, C.R. and I.A. Aksay, Microchannel molding: A soft lithography-inspired approach to micrometer-scale patterning. Journal of Materials Research, 2005. 20(8): p. 1995 - 2003.

25. Faustino, V., et al., Biomedical microfluidic devices by using low-cost fabrication techniques: A review. Journal of Biomechanics.

26. Wu, H., et al., Fabrication of complex threedimensional microchannel systems in PDMS. J Am Chem Soc, 2003. 125(2): p. 554-9.

27. Becker, H. and L.E. Locascio, Polymer microfluidic devices. Talanta, 2002. 56(2): p. 267-287.

28. Bartholomeusz, D.A., R.W. Boutté, and J.D. Andrade, Xurography: rapid prototyping of microstructures using a cutting plotter. IEEE/ASME Journal of Microelectromechanical Systems, 2005. 14(6): p. 1364-1374.

29. Martínez-López, J., et al., Xurography as a rapid fabrication alternative for point-of-care devices: Assessment of passive micromixers. Sensors, 2016. 16(5): p. 705.

30. Neuville, A., et al., Xurography for microfluidics on a reactive solid. Lab on a Chip, 2017. 17(2): p. 293-303. 